\title{
Research Article \\ On Equivalence of Some Iterations Convergence for Quasi-Contraction Maps in Convex Metric Spaces
}

\author{
Zhiqun Xue, ${ }^{1}$ Guiwen Lv, ${ }^{1}$ and B. E. Rhoades ${ }^{2}$ \\ ${ }^{1}$ Department of Mathematics and Physics, Shijiazhuang Railway University, Shijiazhuang 050043, China \\ ${ }^{2}$ Department of Mathematics, Indiana University, Bloomington, IN 47405-7106, USA
}

Correspondence should be addressed to Zhiqun Xue, xuezhiqun@126.com

Received 23 July 2010; Accepted 9 September 2010

Academic Editor: Marlène Frigon

Copyright (C) 2010 Zhiqun Xue et al. This is an open access article distributed under the Creative Commons Attribution License, which permits unrestricted use, distribution, and reproduction in any medium, provided the original work is properly cited.

We show the equivalence of the convergence of Picard and Krasnoselskij, Mann, and Ishikawa iterations for the quasi-contraction mappings in convex metric spaces.

\section{Introduction}

Let $(E, d)$ be a complete metric space and $I=[0,1]$. Denote $E^{2}=E \times E, I^{2}=I \times I$. A continuous mapping $W: E^{2} \times I^{2} \rightarrow E$ is said to be a convex structure on $E$ [1] if for all $u, z_{1}, z_{2} \in$ $E, \lambda_{1}, \lambda_{2} \in I$ with $\lambda_{1}+\lambda_{2}=1$ such that

$$
\begin{gathered}
d\left(u, W\left(z_{1}, z_{2} ; \lambda_{1}, \lambda_{2}\right)\right) \leq \lambda_{1} d\left(u, z_{1}\right)+\lambda_{2} d\left(u, z_{2}\right) ; \\
W\left(z_{1}, z_{2} ; 1,0\right)=z_{1}, \quad W\left(z_{1}, z_{2} ; 0,1\right)=z_{2} .
\end{gathered}
$$

If $(E, d)$ satisfies the conditions of convex structure, then $(E, d)$ is called convex metric space that is denoted as $(E, d, W)$.

In the following part, we will consider a few iteration sequences in convex metric space $(E, d, W)$. Suppose that $T$ is a self-map of $E$.

Picard iteration is as follows:

$$
\forall p_{0} \in E, \quad p_{n+1}=T p_{n}=T^{n+1} p_{0}, \quad n \geq 0 .
$$


Krasnoselskij iteration is as follows:

$$
\forall v_{0} \in E, \quad v_{n+1}=W\left(v_{n}, T v_{n} ; 1-\lambda, \lambda\right), \quad n \geq 0,
$$

where $\lambda \in[0,1]$.

Mann iteration is as follows:

$$
\forall u_{0} \in E, \quad u_{n+1}=W\left(u_{n}, T u_{n} ; 1-a_{n}, a_{n}\right), \quad n \geq 0,
$$

where $a_{n} \in[0,1]$.

Ishikawa iteration is as follows:

$$
\begin{gathered}
\forall x_{0} \in E, \\
x_{n+1}=W\left(x_{n}, T y_{n} ; 1-a_{n}, a_{n}\right), \quad n \geq 0, \\
y_{n}=W\left(x_{n}, T x_{n} ; 1-b_{n}, b_{n}\right), \quad n \geq 0,
\end{gathered}
$$

where $a_{n}, b_{n} \in[0,1]$ for all $n \geq 0$.

A mapping $T: E \rightarrow E$ is called contractive if there exists $L \in(0,1)$ such that

$$
d(T x, T y) \leq L d(x, y)
$$

for all $x, y \in E$.

The map $T$ is called Kannan mapping [2] if there exists $b \in(0,1 / 2)$ such that

$$
d(T x, T y) \leq b[d(x, T x)+d(y, T y)]
$$

for all $x, y \in E$.

A similar definition of mapping is due to the work Chatterjea [3] (that is called Chatterjea mapping), if there exists $c \in(0,1 / 2)$ such that

$$
d(T x, T y) \leq c[d(x, T y)+d(y, T x)]
$$

for all $x, y \in E$.

Combining above three definitions, Zamfirescu [4] showed the following result.

Theorem 1.1. Let $(E, d)$ be a complete metric space and $T: E \rightarrow E$ a mapping for which there exist the real numbers $a, b$, and $c$ satisfying $a \in(0,1), b, c \in(0,1 / 2)$ such that, for any pair $x, y \in E$, at least one of the following conditions holds:

$$
\begin{aligned}
& \text { (z1) } d(T x, T y) \leq a d(x, y) \\
& \text { (z2) } d(T x, T y) \leq b[d(x, T x)+d(y, T y)] \\
& \text { (z3) } d(T x, T y) \leq c[d(x, T y)+d(y, T x)]
\end{aligned}
$$

Then $T$ has a unique fixed point, and the Picard iteration converges to fixed point. This class mapping is called Zamfirescu mapping. 
In 1974, Ćirić [5] introduced one of the most general contraction mappings and obtained that the unique fixed point can be approximated by Picard iteration. This mapping is called quasi-contractive if there exists $\delta \in(0,1)$ such that

$$
d(T x, T y) \leq \delta \cdot \max \{d(x, y), d(x, T x), d(y, T y), d(x, T y), d(y, T x)\},
$$

for any $x, y \in E$.

Clearly, every quasi-contraction mapping is the most general of above mappings.

Later on, in 1992, Xu [6] proved that Ishikawa iteration can also be used to approximate the fixed points of quasi-contraction mappings in real Banach spaces.

Theorem 1.2. Let $C$ be any nonempty closed convex subset of a Banach space $X$ and $T: C \rightarrow C$ a quasi-contraction mapping. Suppose that $\alpha_{n}>0$ for all $n$ and $\sum \alpha_{n}=\infty$. Then the Ishikawa iteration sequence $\left\{x_{n}\right\}$ defined by (1)-(3) converges strongly to the unique fixed point $x^{*}$ of $T$.

In this paper, we will show the equivalence of the convergence of Picard and Krasnoselskij, Mann, and Ishikawa iterations for the quasi-contraction mappings in convex metric spaces.

Lemma 1.3. Let $\left\{\rho_{n}\right\}_{n=0}^{\infty}$ be a nonnegative sequence which satisfies the following inequality

$$
\rho_{n+1} \leq\left(1-\theta_{n}\right) \rho_{n}+\sigma_{n}, \quad n \geq 0,
$$

where $\theta_{n} \in(0,1), \sum_{n=0}^{\infty} \theta_{n}=\infty$, and $\sigma_{n} / \theta_{n} \rightarrow 0$ as $n \rightarrow \infty$. Then $\rho_{n} \rightarrow 0$ as $n \rightarrow \infty$, (see [7]).

\section{Results for Quasi-Contraction Mappings}

Theorem 2.1. Let $(E, d, W)$ be a convex metric space, $T: E \rightarrow E$ a quasi-contraction mapping with $F(T) \neq \emptyset$. Suppose that $\left\{p_{n}\right\}_{n=0}^{\infty},\left\{v_{n}\right\}_{n=0}^{\infty}$ are defined by the iterative processes (1.3) and (1.4), respectively. Then, the following two assertions are equivalent:

(i) Picard iteration (1.3) converges strongly to the unique fixed point $q \in F(T)$;

(ii) Krasnoselskij iteration (1.4) converges strongly to the unique fixed point $q \in F(T)$.

Proof. First, we show (i) $\Rightarrow$ (ii), that is, $d\left(p_{n}, q\right) \rightarrow 0$ as $n \rightarrow \infty \Rightarrow d\left(v_{n}, q\right) \rightarrow$ as $n \rightarrow \infty$. From (1.3), (1.4), and (1.1), we can get

$$
\begin{aligned}
d\left(v_{n+1}, p_{n+1}\right) & =d\left(W\left(v_{n}, T v_{n} ; 1-\lambda, \lambda\right), T p_{n}\right) \\
& \leq(1-\lambda) d\left(v_{n}, T p_{n}\right)+\lambda d\left(T v_{n}, T p_{n}\right) \\
& \leq(1-\lambda) d\left(v_{n}, p_{n}\right)+(1-\lambda) d\left(p_{n}, T p_{n}\right)+\lambda d\left(T v_{n}, T p_{n}\right) \\
& \leq(1-\lambda) d\left(v_{n}, p_{n}\right)+\frac{1-\lambda}{1-\delta} d\left(p_{n}, q\right)+\lambda d\left(T v_{n}, T p_{n}\right) .
\end{aligned}
$$


Next, we consider $d\left(T v_{n}, T p_{n}\right)$. Using (1.10) with $x=p_{n}, y=v_{n}$, to obtain

$$
d\left(T v_{n}, T p_{n}\right) \leq \delta \cdot \max \left\{d\left(v_{n}, p_{n}\right), d\left(v_{n}, T v_{n}\right), d\left(p_{n}, T p_{n}\right), d\left(v_{n}, T p_{n}\right), d\left(p_{n}, T v_{n}\right)\right\}
$$

Set

$$
A_{n}=\left\{v_{i}\right\}_{i=0}^{n} \cup\left\{p_{i}\right\}_{i=0}^{n} \cup\left\{T p_{i}\right\}_{i=0}^{n} \cup\left\{T v_{i}\right\}_{i=0}^{n}, \quad \gamma_{n}=\operatorname{diam}\left\{A_{n}\right\}
$$

Then $\left\{A_{n}\right\}$ is bounded. Without loss of generality, we let $\gamma_{n}>0$ for each $n$. Indeed, we will show this conclusion from the some following cases.

Case 1. Let $\gamma_{n}=d\left(T p_{i}, T v_{j}\right)$ for some $0 \leq i, j \leq n$. Then, from (1.10) and the above $\gamma_{n}$, we have

$$
\begin{aligned}
\gamma_{n} & =d\left(T p_{i}, T v_{j}\right) \\
& \leq \delta \cdot \max \left\{d\left(p_{i}, v_{j}\right), d\left(v_{j}, T v_{j}\right), d\left(p_{i}, T p_{i}\right), d\left(v_{j}, T p_{i}\right), d\left(p_{i}, T v_{j}\right)\right\} \\
& \leq \delta \gamma_{n}<\gamma_{n},
\end{aligned}
$$

and it leads to a contradiction. Thus, $\gamma_{n} \neq d\left(T p_{i}, T v_{j}\right)$. Similarity to $\gamma_{n}=d\left(T p_{i}, T p_{j}\right)$ or $\gamma_{n}=$ $d\left(T v_{i}, T v_{j}\right)$ is also impossible.

Case 2. Let $\gamma_{n}=d\left(p_{i}, v_{j}\right)$ for some $0 \leq i, j \leq n$.

(i) If $j=0$, then $\gamma_{n}=d\left(p_{i}, v_{0}\right)$.

(ii) If $j \geq 1, i=0$, then, from (1.4) and (1.1)

$$
\begin{aligned}
\gamma_{n} & =d\left(p_{0}, v_{j}\right) \\
& =d\left(p_{0}, W\left(v_{j-1}, T v_{j-1} ; 1-\lambda, \lambda\right)\right) \\
& \leq(1-\lambda) d\left(p_{0}, v_{j-1}\right)+\lambda d\left(p_{0}, T v_{j-1}\right) \\
& \leq(1-\lambda) d\left(p_{0}, v_{j-1}\right)+\lambda \gamma_{n},
\end{aligned}
$$

that is, $\gamma_{n}=d\left(p_{0}, v_{j-1}\right)$. By induction on $j$, we can obtain $\gamma_{n}=d\left(p_{0}, v_{0}\right)$.

(iii) If $j \geq 1, i \geq 1$, from (1.4) and (1.1)

$$
\begin{aligned}
\gamma_{n} & =d\left(p_{i}, v_{j}\right) \\
& =d\left(p_{i}, W\left(v_{j-1}, T v_{j-1} ; 1-\lambda, \lambda\right)\right) \\
& \leq(1-\lambda) d\left(p_{i}, v_{j-1}\right)+\lambda d\left(p_{i}, T v_{j-1}\right) \\
& \leq(1-\lambda) d\left(p_{i}, v_{j-1}\right)+\lambda \gamma_{n},
\end{aligned}
$$

it implies that $\gamma_{n}=d\left(p_{i}, v_{j-1}\right)$. By induction on $j$, we can get $\gamma_{n}=d\left(p_{i}, v_{0}\right)$. 
Case 3. Let $\gamma_{n}=d\left(v_{i}, v_{j}\right)$ for some $0 \leq i, j \leq n$. Without loss of generality, we set $0 \leq i<j \leq n$. Then, from (1.4), (1.1)

$$
\begin{aligned}
\gamma_{n} & =d\left(v_{i}, v_{j}\right) \\
& \leq \mathrm{d}\left(v_{i}, W\left(v_{j-1}, T v_{j-1} ; 1-\lambda, \lambda\right)\right) \\
& \leq(1-\lambda) d\left(v_{i}, v_{j-1}\right)+\lambda d\left(v_{i}, T v_{j-1}\right) \\
& \leq(1-\lambda) d\left(v_{i}, v_{j-1}\right)+\lambda \gamma_{n},
\end{aligned}
$$

it implies that $\gamma_{n}=d\left(v_{i}, v_{j-1}\right)$, and by induction on $j$, we may get $\gamma_{n}=d\left(v_{i}, v_{i}\right)=0$, which is a contradiction.

Case 4. Let $\gamma_{n}=d\left(v_{i}, T p_{j}\right)$ for some $0 \leq i, j \leq n$.

(i) If $i=0$, then $\gamma_{n}=d\left(v_{0}, T p_{j}\right)$.

(ii) If $i \geq 1$, from (1.4), (1.1), then

$$
\begin{aligned}
\gamma_{n}= & d\left(v_{i}, T p_{j}\right) \\
\leq & d\left(W\left(v_{i-1}, T v_{i-1} ; 1-\lambda, \lambda\right), T p_{j}\right) \\
\leq & (1-\lambda) d\left(v_{i-1}, T p_{j}\right)+\lambda d\left(T v_{i-1}, T p_{j}\right) \\
\leq & (1-\lambda) d\left(v_{i-1}, T p_{j}\right) \\
& +\lambda \delta \cdot \max \left\{d\left(v_{i-1}, p_{j}\right), d\left(v_{i-1}, T v_{i-1}\right), d\left(p_{j}, T p_{j}\right), d\left(v_{i-1}, T p_{j}\right), d\left(p_{j}, T v_{i-1}\right)\right\} \\
\leq & (1-\lambda) d\left(v_{i-1}, T p_{j}\right)+\lambda \delta \gamma_{n} \\
\leq & (1-\lambda) d\left(v_{i-1}, T p_{j}\right)+\lambda \gamma_{n}
\end{aligned}
$$

it implies that $\gamma_{n}=d\left(v_{i-1}, T p_{j}\right)$ and by induction on $i$, then $\gamma_{n}=d\left(v_{0}, T p_{j}\right)$.

Case 5. Let $\gamma_{n}=\mathrm{d}\left(p_{i}, T v_{j}\right)$ for some $0 \leq i, j \leq n$.

(i) If $i=0$, then $\gamma_{n}=d\left(p_{0}, T v_{j}\right)$.

(ii) If $i \geq 1$, then, from (1.3) and (1.10)

$$
\begin{aligned}
\gamma_{n} & =d\left(p_{i}, T v_{j}\right) \\
& \leq d\left(T p_{i-1}, T v_{j}\right) \\
& \leq \lambda \delta \cdot \max \left\{d\left(p_{i-1}, v_{j}\right), d\left(p_{i-1}, T p_{i-1}\right), d\left(v_{j}, T v_{j}\right), d\left(p_{i-1}, T v_{j}\right), d\left(v_{j}, T \mathrm{p}_{i-1}\right)\right\} \\
& \leq \lambda \delta \gamma_{n},
\end{aligned}
$$

this is a contradiction. 
Case 6. let $\gamma_{n}=d\left(v_{i}, T v_{j}\right)$ for some $0 \leq i, j \leq n$.

(i) If $i=0$, then $\gamma_{n}=d\left(v_{0}, T v_{j}\right)$.

(ii) If $i \geq 1$, then, from (1.4) and (1.10)

$$
\begin{aligned}
\gamma_{n}= & d\left(v_{i}, T v_{j}\right) \\
\leq & d\left(W\left(v_{i-1}, T v_{i-1} ; 1-\lambda, \lambda\right), T v_{j}\right) \\
\leq & (1-\lambda) d\left(v_{i-1}, T v_{j}\right)+\lambda d\left(T v_{i-1}, T v_{j}\right) \\
\leq & (1-\lambda) d\left(v_{i-1}, T v_{j}\right) \\
& +\lambda \delta \cdot \max \left\{d\left(v_{i-1}, v_{j}\right), d\left(v_{i-1}, T v_{i-1}\right), d\left(v_{j}, T v_{j}\right), d\left(v_{i-1}, T v_{j}\right), d\left(v_{j}, T v_{i-1}\right)\right\} \\
\leq & (1-\lambda) d\left(v_{i-1}, T v_{j}\right)+\lambda \delta \gamma_{n},
\end{aligned}
$$

it implies that $\gamma_{n}=d\left(v_{0}, T v_{j}\right)$.

Case 7. Let $\gamma_{n}=d\left(p_{i}, p_{j}\right)$ for some $0 \leq i, j \leq n$.

(i) If $i=0, j>0$, then $\gamma_{n}=d\left(p_{0}, p_{j}\right)$.

(ii) If $i, j \geq 1$, then, from (1.3), (1.10)

$$
\begin{aligned}
\gamma_{n} & =d\left(p_{i}, p_{j}\right) \\
& \leq d\left(T p_{i-1}, T p_{j-1}\right) \\
& \leq \delta \cdot \max \left\{d\left(p_{i-1}, p_{j-1}\right), d\left(p_{i-1}, T p_{i-1}\right), d\left(p_{j-1}, T p_{j-1}\right), d\left(p_{i-1}, T p_{j-1}\right), d\left(\mathrm{p}_{j-1}, T p_{i-1}\right)\right\} \\
& \leq \delta \gamma_{n},
\end{aligned}
$$

it is a contradiction.

Case 8. let $\gamma_{n}=d\left(p_{i}, T p_{j}\right)$ for some $0 \leq i, j \leq n$.

(i) If $i=0$, then $\gamma_{n}=d\left(p_{0}, T p_{j}\right)$.

(ii) If $i \geq 1$, then, from (1.3) and (1.10)

$$
\begin{aligned}
\gamma_{n} & =d\left(p_{i}, T p_{j}\right) \\
& \leq d\left(T p_{i-1}, T p_{j}\right) \\
& \leq \delta \cdot \max \left\{d\left(p_{i-1}, p_{j}\right), d\left(p_{i-1}, T p_{i-1}\right), d\left(p_{j}, T p_{j}\right), d\left(p_{i-1}, T p_{j}\right), d\left(p_{j}, T p_{i-1}\right)\right\} \\
& \leq \delta \gamma_{n},
\end{aligned}
$$

which is a contradiction. 
Fixed Point Theory and Applications

Set

$$
\begin{aligned}
\eta_{n}=\max \{ & \max \left\{d\left(p_{i}, v_{0}\right): 0<i \leq n\right\}, \max \left\{d\left(v_{0}, T p_{i}\right): 0<i \leq n\right\}, \\
& \max \left\{d\left(v_{0}, T v_{i}\right): 0<i \leq n\right\}, \max \left\{d\left(p_{0}, p_{i}\right): 0<i \leq n\right\}, \\
& \left.\max \left\{d\left(p_{0}, T v_{i}\right): 0<i \leq n\right\}, \max \left\{d\left(p_{0}, T p_{i}\right): 0<i \leq n\right\}, M\right\},
\end{aligned}
$$

where $M=\max \left\{d\left(p_{0}, v_{0}\right), d\left(v_{0}, T p_{0}\right), d\left(v_{0}, T v_{0}\right), d\left(p_{0}, T v_{0}\right), d\left(p_{0}, T p_{0}\right)\right\}$.

In view of the above cases, then $\gamma_{n}=\eta_{n}$, and we obtain that $\left\{\gamma_{n}\right\}$ is bounded.

Indeed, suppose that $\gamma_{n}=d\left(p_{i}, v_{0}\right)$ for some $0<i \leq n$. Then,

$$
\begin{aligned}
\gamma_{n}= & d\left(p_{i}, v_{0}\right) \\
\leq & d\left(p_{i}, T v_{0}\right)+d\left(T v_{0}, v_{0}\right) \\
= & d\left(T p_{i-1}, T v_{0}\right)+d\left(T v_{0}, v_{0}\right) \\
\leq & d\left(T p_{i-1}, T v_{0}\right)+d\left(T v_{0}, v_{0}\right) \\
\leq & \delta \cdot \max \left\{d\left(p_{i-1}, v_{0}\right), d\left(v_{0}, T v_{0}\right), d\left(p_{i-1}, T p_{i-1}\right), d\left(v_{0}, T p_{i-1}\right), d\left(p_{i-1}, T v_{0}\right)\right\} \\
& +d\left(T v_{0}, v_{0}\right) \\
\leq & \delta \gamma_{n}+d\left(T v_{0}, v_{0}\right),
\end{aligned}
$$

which implies that $\gamma_{n} \leq(1 /(1-\delta)) d\left(T v_{0}, v_{0}\right)$. Similarly, if $\gamma_{n}=d\left(v_{0}, T p_{i}\right)$ or $\gamma_{n}=d\left(v_{0}, T v_{i}\right)$, we also obtain $\gamma_{n} \leq(1 /(1-\delta)) d\left(T v_{0}, v_{0}\right)$.

On the other hand, suppose that $\gamma_{n}=d\left(p_{0}, p_{i}\right)$ for some $0<i \leq n$. Then,

$$
\begin{aligned}
\gamma_{n}= & d\left(p_{0}, p_{i}\right) \\
\leq & d\left(p_{0}, T p_{0}\right)+d\left(T p_{0}, T p_{i-1}\right) \\
\leq & d\left(p_{0}, T p_{0}\right) \\
& +\delta \cdot \max \left\{d\left(p_{0}, p_{i-1}\right), d\left(p_{0}, T p_{0}\right), d\left(p_{i-1}, T p_{i-1}\right), d\left(p_{0}, T p_{i-1}\right), d\left(p_{i-1}, T p_{0}\right)\right\} \\
\leq & d\left(p_{0}, T p_{0}\right)+\delta \gamma_{n},
\end{aligned}
$$

which implies that $\gamma_{n} \leq(1 /(1-\delta)) d\left(T p_{0}, p_{0}\right)$. Similarly, if $\gamma_{n}=d\left(p_{0}, T v_{i}\right)$ or $\gamma_{n}=d\left(p_{0}, T p_{i}\right)$, we also obtain $\gamma_{n} \leq(1 /(1-\delta)) d\left(T p_{0}, p_{0}\right)$. Therefore, from the above results, we obtain that $\gamma_{n} \leq(1 /(1-\delta)) M$, that is, $\left\{A_{n}\right\}$ is bounded.

For each $n \in \mathbb{N}$, define

$$
B_{n}=\left\{v_{i}\right\}_{i \geq n} \cup\left\{p_{i}\right\}_{i \geq n} \cup\left\{T p_{i}\right\}_{i \geq n} \cup\left\{T v_{i}\right\}_{i \geq n}, \quad R_{n}=\operatorname{diam}\left(B_{n}\right) .
$$


Then, using the same proof above, it can be shown that

$$
\begin{aligned}
R_{n}=\operatorname{diam}\left(B_{n}\right)=\max \{ & \sup \left\{d\left(p_{i}, v_{n}\right): i \geq n\right\}, \sup \left\{d\left(v_{n}, T p_{i}\right): i \geq n\right\}, \\
& \sup \left\{d\left(p_{n}, T v_{i}\right): i \geq n\right\}, \sup \left\{d\left(v_{n}, T v_{i}\right): i \geq n\right\}, \\
& \left.\sup \left\{d\left(p_{n}, p_{i}\right): i>n\right\}, \sup \left\{d\left(p_{n}, T p_{i}\right): i \geq n\right\}\right\} .
\end{aligned}
$$

If $R_{n}=\sup \left\{d\left(p_{i}, v_{n}\right): i \geq n\right\}$, and using (1.1) and (1.4), then

$$
\begin{aligned}
R_{n} & =\sup _{i \geq n} d\left(p_{i}, v_{n}\right) \\
& =\sup _{i \geq n} d\left(p_{i}, W\left(v_{n-1}, T v_{n-1} ; 1-\lambda, \lambda\right)\right) \\
& \leq \sup _{i \geq n}\left\{(1-\lambda) d\left(p_{i}, v_{n-1}\right)+\lambda d\left(p_{i}, T v_{n-1}\right)\right\} \\
& \leq \sup _{i \geq n}\left\{(1-\lambda) R_{n-1}+\lambda d\left(T p_{i-1}, T v_{n-1}\right)\right\} \\
& \leq \sup _{i \geq n}\left\{(1-\lambda) R_{n-1}+\lambda \delta\right. \\
& \leq(1-\lambda) R_{n-1}+\lambda \delta R_{n-1} \\
& =(1-\lambda(1-\delta)) R_{n-1} \\
& \leq \cdots \\
& \leq(1-\lambda(1-\delta))^{n} R_{0} \\
& \longrightarrow 0
\end{aligned}
$$

as $n \rightarrow \infty$. Since $d\left(T v_{n}, T p_{n}\right) \leq R_{n}$, hence $d\left(T v_{n}, T p_{n}\right) \rightarrow 0$ as $n \rightarrow \infty$. Similarly, if $R_{n}=$ $\sup \left\{d\left(v_{n}, T p_{i}\right): i \geq n\right\}$ or $R_{n}=\sup \left\{d\left(v_{n}, T v_{i}\right): i \geq n\right\}, R_{n}=\sup \left\{d\left(p_{n}, T v_{i}\right): i \geq n\right\}, R_{n}=$ $\sup \left\{d\left(v_{n}, T v_{i}\right): i \geq n\right\}, R_{n}=\sup \left\{d\left(p_{n}, p_{i}\right): i>n\right\}, R_{n}=\sup \left\{d\left(p_{n}, T p_{i}\right): i \geq n\right\}$, we may obtain the similar results. Therefore, from (2.1), we get

$$
d\left(v_{n+1}, p_{n+1}\right) \leq(1-\lambda) d\left(v_{n}, p_{n}\right)+\sigma_{n}
$$

where $\sigma_{n}=((1-\lambda) /(1-\delta)) d\left(p_{n}, q\right)+\lambda d\left(T v_{n}, T p_{n}\right)$.

In (2.19), set $\rho_{n}=d\left(v_{n}, p_{n}\right)$. Then (2.19) is as follows:

$$
\rho_{n+1} \leq(1-\lambda) \rho_{n}+\sigma_{n}
$$

By Lemma 1.3, we have $d\left(v_{n}, p_{n}\right) \rightarrow 0$ as $n \rightarrow \infty$. From the inequality $0 \leq d\left(v_{n}, q\right) \leq$ $d\left(v_{n}, p_{n}\right)+d\left(p_{n}, q\right)$, we have $\lim _{n \rightarrow \infty} d\left(v_{n}, q\right)=0$.

Conversely, we will prove that (ii) $\Rightarrow$ (i). If $\lambda=1$, then $v_{n+1}=W\left(v_{n}, T v_{n} ; 0,1\right)=T v_{n}$ is Picard iteration. 
Theorem 2.2. Let $(E, d, W), T, F(T)$ be as in Theorem 2.1. Suppose that $\left\{u_{n}\right\}_{n=0}^{\infty},\left\{x_{n}\right\}_{n=0}^{\infty}$ are defined by the iterative processes (1.5) and (1.6), respectively, and $\left\{a_{n}\right\}_{n=0}^{\infty},\left\{b_{n}\right\}_{n=0}^{\infty}$ are real sequences in $[0,1]$ such that $\sum_{n=0}^{\infty} a_{n}=\infty$. Then, the following two assertions are equivalent:

(i) Mann iteration (1.5) converges strongly to the unique fixed point $q \in F(T)$;

(ii) Ishikawa iteration (1.6) converges strongly to the unique fixed point $q \in F(T)$.

Proof. If the Ishikawa iteration (1.6) converges strongly to $q$, then setting $b_{n}=0$, for all $n \geq 0$, in (1.6), we can get the convergence of Mann iteration (1.5). Conversely, we will show that (i) $\Rightarrow$ (ii). Letting $\lim _{n \rightarrow \infty} d\left(u_{n}, q\right)=0$, we want to prove $\lim _{n \rightarrow \infty} d\left(x_{n}, q\right)=0$.

From (1.5) and (1.6),

$$
\begin{aligned}
d\left(x_{n+1},\right. & \left.u_{n+1}\right) \\
= & d\left(W\left(x_{n}, T y_{n} ; 1-a_{n}, a_{n}\right), W\left(u_{n}, T u_{n} ; 1-a_{n}, a_{n}\right)\right) \\
\leq & \left(1-a_{n}\right) d\left(x_{n}, W\left(u_{n}, T u_{n} ; 1-a_{n}, a_{n}\right)\right)+a_{n} d\left(T y_{n}, W\left(u_{n}, T u_{n} ; 1-a_{n}, a_{n}\right)\right) \\
\leq & \left(1-a_{n}\right)^{2} d\left(x_{n}, u_{n}\right)+a_{n}\left(1-a_{n}\right) d\left(x_{n}, T u_{n}\right) \\
& +\left(1-a_{n}\right) a_{n} d\left(T y_{n}, u_{n}\right)+a_{n}^{2} d\left(T y_{n}, T u_{n}\right) \\
\leq & \left(1-a_{n}\right)^{2} d\left(x_{n}, u_{n}\right)+a_{n}\left(1-a_{n}\right) d\left(x_{n}, u_{n}\right)+a_{n}\left(1-a_{n}\right) d\left(u_{n}, T u_{n}\right) \\
& +\left(1-a_{n}\right) a_{n} d\left(T y_{n}, T u_{n}\right)+\left(1-a_{n}\right) a_{n} d\left(T u_{n}, u_{n}\right)+a_{n}^{2} d\left(T y_{n}, T u_{n}\right) \\
= & \left(1-a_{n}\right) d\left(x_{n}, u_{n}\right)+2 a_{n}\left(1-a_{n}\right) d\left(u_{n}, T u_{n}\right)+a_{n} d\left(T y_{n}, T u_{n}\right) \\
\leq & \left(1-a_{n}\right) d\left(x_{n}, u_{n}\right)+2 a_{n}\left(1-a_{n}\right) d\left(u_{n}, q\right) \\
& +2 a_{n}\left(1-a_{n}\right) d\left(T u_{n}, T q\right)+a_{n} d\left(T y_{n}, T u_{n}\right) \\
\leq & \left(1-a_{n}\right) d\left(x_{n}, u_{n}\right)+2 a_{n} \frac{1-a_{n}}{1-\delta} d\left(u_{n}, q\right)+a_{n} d\left(T y_{n}, T u_{n}\right) .
\end{aligned}
$$

Using (1.10) with $x=y_{n}, y=u_{n}$, to obtain

$$
d\left(T y_{n}, T u_{n}\right) \leq \delta \cdot \max \left\{d\left(y_{n}, u_{n}\right), d\left(u_{n}, T u_{n}\right), d\left(y_{n}, T y_{n}\right), d\left(y_{n}, T u_{n}\right), d\left(u_{n}, T y_{n}\right)\right\}
$$

set

$$
\begin{gathered}
A_{n n}=\left\{u_{i}\right\}_{i=0}^{n} \cup\left\{y_{i}\right\}_{i=0}^{n} \cup\left\{x_{i}\right\}_{i=0}^{n} \cup\left\{T u_{i}\right\}_{i=0}^{n} \cup\left\{T y_{i}\right\}_{i=0}^{n} \cup\left\{T x_{i}\right\}_{i=0}^{n}, \\
\gamma_{n n}=\operatorname{diam}\left(A_{n n}\right) .
\end{gathered}
$$

Applying the similar proof methods of Theorem 2.1, we obtain that $\left\{A_{n n}\right\}$ is also bounded. The other proof is the same as that of Theorem 2.1 and is here omitted. 


\section{Acknowledgments}

The authors are extremely grateful to Professor B. E. Rhoades of Indiana University for providing useful information and many help. They also thank the referees for their valuable comments and suggestions.

\section{References}

[1] W. Takahashi, "A convexity in metric space and nonexpansive mappings. I," Kōdai Mathematical Seminar Reports, vol. 22, pp. 142-149, 1970.

[2] R. Kannan, "Some results on fixed points," Bulletin of the Calcutta Mathematical Society, vol. 60, pp. 71-76, 1968.

[3] S. K. Chatterjea, "Fixed-point theorems," Comptes Rendus de l'Académie Bulgare des Sciences, vol. 25, pp. 727-730, 1972.

[4] T. Zamfirescu, "Fix point theorems in metric spaces," Archiv der Mathematik, vol. 23, pp. 292-298, 1972.

[5] L. B. Cirić, "A generalization of Banach's contraction principle," Proceedings of the American Mathematical Society, vol. 45, pp. 267-273, 1974

[6] H. K. Xu, "A note on the Ishikawa iteration scheme," Journal of Mathematical Analysis and Applications, vol. 167 , no. 2 , pp. 582-587, 1992.

[7] X. Weng, "Fixed point iteration for local strictly pseudo-contractive mapping," Proceedings of the American Mathematical Society, vol. 113, no. 3, pp. 727-731, 1991. 